\title{
Inferring relationships between temperature, bulk composition and wave speeds using Mixture Density Networks
}

\author{
ASHIM RIJAL, LAURA COBDEN, JEANNOT TRAMPERT \\ AND CARLO MANS \\ Utrecht University \\ Presenting Author: a.rijal@uu.nl
}

The study of seismic data provides vital information on the internal structure of the Earth. Information such as variation of wave speeds has shown the presence of seismically distinct structures (e.g. LLSVPs) in the Earth's mantle. In order to determine the thermochemical properties and dynamic relevance of these structures, it is crucial to understand the relationship between wave speeds and temperature/composition. The relationship becomes non-linear when we include multiple physical properties and hence, a simple linear scaling to go from seismic observables to thermal or chemical structure may be inappropriate. In this study we use a neural network based approach to implicitly learn the non-linear mapping between temperature, bulk composition (expressed in terms of 6 oxides: $\mathrm{SiO} 2, \mathrm{MgO}, \mathrm{Al} 2 \mathrm{O} 3, \mathrm{FeO}, \mathrm{Na} 2 \mathrm{O}, \mathrm{CaO}$ ) and wave speeds. Wave speeds are calculated for 1000 randomly-chosen bulk compositions at lower mantle temperatures and pressures using thermodynamic modelling software. We train the neural network using wave speeds and pressure (depth) as the input, and temperature and composition as target outputs. The network then approximates probability density functions for temperature and composition, for a given input of P- and S-wave speeds. The posterior probability density function allows us to interpret seismic tomography models in terms of physical parameters, crucially, with uncertainties on the inferred bulk compositions and temperatures. We further investigate the joint probability density function between different oxides (and temperature) to account for any possible trade-off/correlation between parameters. 